\section{GAMELPP: A PASCAL program for solving two-person zero-sum games on a microcomputer}

\section{JERRY M. MANHEIMER \\ University of New Mexico, Albuquerque, New Mexico 87131}

The solution of a two-person zero-sum game provides optimal strategies for both players. In game-theoretic terms, the solution specifies a minimax strategy for one player and a maximin strategy for the other. These strategies can be pure strategies, that is, single strategies selected from the set of strategies available to each player, or they may be mixed strategies that are weighted composites of the single strategies. The weights in these composites consist of probabilities for playing the various strategies. The probabilities in a pair of mixed strategies are selected so that the expected value of the game is optimal for each player. The solution of a two-person zero-sum game, therefore, rests on finding a vector of probabilities $x$ that would allow one player to maximize the game value and another vector of probabilities $y$ enabling the other player to minimize the game value.

Solutions consisting of pure strategies can be readily obtained by examination of the game matrix, and the mixed strategies in a 2 by 2 game matrix can be determined by applying straightforward computational formulas (e.g., Coombs, Dawes, \& Tversky, 1970). However, to obtain mixed-strategy solutions for game matrices with dimensionality greater than two, linear programming is required (Luce \& Raiffa, 1957). Linear programming is a technique for maximizing and minimizing linear functions that are subject to a number of linear constraints and nonnegativity conditions. Since the value of a game can easily be expressed as a linear function, and the rows and columns of a game matrix can serve as constraints, linear programming may be utilized to determine the vectors of probabilities $x$ and $y$ that, respectively, maximize and minimize the value of a two-person zero-sum game.

Although linear programming computer programs have been available for some time, they are typically written for general linear programming applications. Many of the programs (such as MPOS, ${ }^{1}$ for example) are large "canned" packages capable of solving a wide variety of linear programming problems. Since the primary application of linear programming in psychology is for solving zero-sum games, the rationale for implementing a large general-purpose "optimization" package for solving a small class of problems is questionable. These general programs require manual conversion of a game problem to a linear programming problem and manual conversion of a linear programming solution back to a game solution. Since linear programming is frequently used in business applications, many programs result in output that contains terminology specific to business problems. This implies that the user would have to separate the information that is relevant to a game solution from information that is irrelevant.

The GAMELPP program was written to address the above problems. Another objective underlying the design of the program was to implement a game program on a microcomputer. Since game matrices are small relative to the matrices typically dealt with by programs on large computers, the advantages of a microcomputer implementation can be realized without a substantial reduction in the range of meaningful problems that can be solved.

Description of Algorithm Employed. The GAMELPP program adopts the convention that the row player in a two-person zero-sum game seeks to maximize the expected value of the game and the column player desires to minimize the game value. The simplex linear programming algorithm (Dantzig, 1963) is then employed to find mixed strategies for each player. The first stage of the simplex algorithm requires the addition of vectors of slack variables to the columns of the game matrix. These variables serve to transform the rows of the matrix from inequalities to equalities. The next stage of the algorithm involves inverting different submatrices of the augmented game matrix until the function of interest attains its maximum or minimum value. The selection of a submatrix for inversion is based on straightforward criteria.

Although the column player wants to minimize the value of the game and the row player desires to maximize the game value, the program maximizes functions for the column player and minimizes functions for the row player. Using the notation of Luce and Raiffa (1952), if $y_{j}$ is the probability of the column player's selecting Strategy $j$ and $x_{i}$ is the row player's probability for selecting Strategy $i$, then letting $V=$ the value of the game, $w_{j}=y_{j} / V$, and $u_{i}=x_{i} / V$, the column player's task is to maximize

$$
w_{1}+w_{2}+w_{3}+\ldots+w_{m}=1 / V
$$

and the row player's task is to minimize

$$
u_{1}+u_{2}+u_{3}+\ldots+u_{n}=1 / V .
$$

The column player wants to maximize Equation 1 since if $1 / V$ is a maximum, then $V$ is a minimum. The row player would hope to minimize Equation 2 since a minimum value for $1 / \mathrm{V}$ assures a maximum value of $\mathrm{V}$.

The maximization problem is easily solved by the simplex algorithm. The minimization problem, however, introduces complications beyond those encountered in the maximization case. To minimize Equation 2, addi. tional vectors consisting of artificial variables must be 
added to the augmented game matrix in addition to the slack variables. To successfully conduct the simplex algorithm, the artificial variables must be successively excluded from the submatrices selected for inversion. The necessity of eliminating artificial variables first before an optimal solution can be obtained requires use of the two-phase simplex algorithm (Swanson, 1980). The two-phase algorithm is employed in GAMELPP to minimize functions and thereby determine optimal strategies for row players.

Program Description. GAMELPP is written in UCSD PASCAL ${ }^{2}$ a version of PASCAL specifically designed for microcomputers, and implemented on a Terak 8510/A microcomputer with a memory capacity of $64 \mathrm{~KB}$. The program can be modified easily for use on other computers, since the only UCSD PASCAL intrinsic used in the program is the STRING data type used to read option statements from a file. The STRINGs can be replaced by packed arrays of characters with little difficulty.

GAMELPP is written in a block-structured programming style. The procedure SOLVE is called once to find the column player's mixed strategy and again for determining the row player's mixed strategy. A number of procedures are embedded in SOLVE and include the logic for carrying out the simplex algorithm. Matrix inversion is accomplished by the ROWOPERATION procedure, which conducts elementary row operations on the augmented game matrix. A number of procedures outside SOLVE and ROWOPERATION take care of the input and output operations.

The game matrix and option requests are specified in a file before program execution. With options, the user may request solutions for the row player, column player, or both players, receive output that displays the augmented matrix ("simplex tableau") for each iteration of the simplex algorithm or only for the initial and final stages of the solution, and send output to the console, printer, or file. Regardless of the option selected, the program output lists the rank of the game matrix, initial and final augmented matrices, the value of the game, and the optimal vector of probabilities for the player selected. If the game is not solved in $\mathbf{n}$ iterations ( $\mathrm{n}$ being determined by the dimensions of the game matrix) or the game matrix cannot be solved by the simplex algorithm, the program stops and an error message is printed.

Due to the small memory capacity of microcomputers, the array that holds the values of the game matrix in GAMELPP is dimensioned to store a 40 by 40 matrix of real numbers. Since the dimensionality of an initial game matrix is increased by the addition of slack and artificial variables, the program can solve game matrices that contain a maximum of 10 strategies for the column player and 19 strategies for the row player. If only the column player's solution is desired, a matrix with 19 strategies/player can be solved.

Availability. Source and output listings can be obtained from the author at no cost: Jerry Manheimer, Department of Psychology, University of New Mexico, Albuquerque, New Mexico 87131.

\section{REFERENCES}

Cohen, C., \& STE IN, J. Multi-purpose optimization system-User's guide. In L. W. Swanson (Ed.), Linear programming: Basic theory and applications. New York: McGraw-Hill, 1980.

Coombs, C. H., Dawes, R. M., \& TVersky, A. Mathematical psychology: An elementary introduction. Englewood Cliffs, N.J: Prentice-Hall, 1970.

Dantzia, O. B. Linear programming and extensions. Princeton, N.J: Princeton University Press, 1963.

Luce, R. D., \& RAIrFA, H. Games and decisions. New York: Wiley, 1957.

Swanson, L. W. Linear programming: Basic theory and applications. New York: McGraw-Hill, 1980.

\section{NOTES}

1. Multipurpose optimization system (see Cohen \& Stein, 1980).

2. Copyright 1978 by the Regents of the University of California (San Diego).

(Accepted for publication May 7, 1982.) 